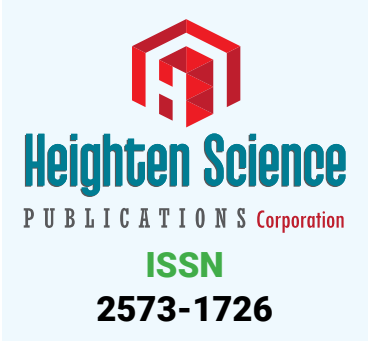

*Address for Correspondence: Zehra Guchan Topcu, Eastern Mediterranean University, Faculty of Health Sciences, Department of Physiotherapy and Rehabilitation, Famagusta, Cyprus, Tel: 009039263030 67; 009054287336 77; 00 903926303067 (extension: 2496); Email: zehraguchan@hotmail.com; zehra.guchan@emu.edu.tr

Submitted: 12 December 2018 Approved: 19 December 2018 Published: 20 December 2018

Copyright: @ 2018 Topcu ZG, et al. This is an open access article distributed under the Creative Commons Attribution License, which permits unrestricted use, distribution, and reproduction in any medium, provided the original work is properly cited

Keywords: Genetics; Syndrome; Tetrasomy; Physiotherapy

Check for updates
Research Article

\section{The effects of physiotherapy and rehabilitation on Pallister-Killian Syndrome: A case report}

\author{
Zehra Guchan Topcu $^{1 *}$ and Selma Uzuner ${ }^{2}$ \\ ${ }^{1}$ Lecturer, Eastern Mediterranean University, Famagusta, Cyprus \\ ${ }^{2}$ Lecturer, University of Kyrenia, Kyrenia, Cyprus
}

\section{Abstract}

The aim of the paper is to determine the effects of physiotherapy and rehabilitation (PHTR) on a child with Pallister-Killian Syndrome (PKS). Gross Motor Function Measure (GMFM) and duration of grasping ping pong ball were the primary outcome measures which were conducted at the beginning, $12^{\text {th }}$ and $24^{\text {th }}$ month. Neurodevelopmental Therapy and Sensory Integration Therapy were used as intervention methods. The most increase with around $50 \%$ was acquired in Section $\mathrm{A}$ of the GMFM. Duration of holding a ping pong ball gradually increased. As a result, this is the first paper presenting a PHTR shedule and its results in a child with PKS.

\section{Introduction}

Pallister-Killian syndrome (PKS), is a rare and sporadic disease caused by the mosaic tetrasomy of the short arm of chromosome 12 . In addition to the two normal copies of chromosome 12, this is related to the presence of an isochromosome $12 \mathrm{p}$ [1]. Although molecular reason of the disease has been determined, the mechanisms caused to this molecular impairment are not clear [2].

All body systems may be affected in PKS $[3,4]$. Severe intellectual disability, motor development problems, deaf, epileptic attacks, hypoplastic lungs, speech delays are some clinical symptoms. These children also have characteristic face features which are abnormal pigmentation lines, specific forehead with temporal hair loss, hypertelorism, short nose and wide mouth $[2,3,5]$.

The first remarkable symptom which clinician doubts is hypotonia which can be severe to affect digestive and respiratory systems [2,5]. Hypotonia continues during growth of these children and this causes difficulties in sitting, standing and moving. Neurological prognosis of these patients becomes more severe with limited motor skills [5].

Thus, there are various factors which affect the development of children with PKS. Physiotherapy and Rehabilitation (PHTR) seems to be essential to prepare the muscles for their normal motor development. To our knowledge, no study has reported a PHTR schedule for the patients with PKS and its effects on them. As a result, this paper aimed to investigate the effects of regularly applied PHTR approaches on a case with PKS.

\section{History}

The child was 3 years old boy. He was born at 37-week of gestation and no prenatal or natal risk factor generated so he was not taken to neonatal incubator. After birth, 
he was treated since bilirubin level increased. Birth weight, birth height, and head circumference were recorded as $2.640 \mathrm{gr}, 47 \mathrm{~cm}$, and $31 \mathrm{~cm}$, respectively. Pediatrician directs the parent to pediatric neurolog with the doubt of a motor disorder. He was diagnosed as PKS at around 9 months. He also had strabismus and very severe level of mental disability. There were hyper and hypo sensitive regions.

\section{Materials and Methods}

An informed consent was signed by the parent. Assessments and treatments were conducted in the Motor Development Unite of the Health Sciences Faculty, Eastern Mediterranean University. The assessments were conducted at the beginning of the treatment $\left(\mathrm{T}_{0}\right), 12$ th month $\left(\mathrm{T}_{1}\right)$, and $24^{\text {th }}$ month $\left(\mathrm{T}_{2}\right)$. Treatment started in July 2015 and last assessment was done in July 2017.

Gross Motor Function Measure (GMFM-88) and duration of grasping ping pong ball were recorded to determine objective results of his motor level. GMFM-88 measures gross motor functions of children in five domains which are lying and rolling, crawling and kneeling, sitting, standing and walk-run-jumping activities. The amount of completion of the movements in the form is scored [6]. Ping pang ball was also used to evaluate the fine motor skill of the case as this is a specific ball type which is commonly used to measure different aspects of motor skills [7].

Interventions

A structured and individualized PHTR schedule was prepared according to the comprehensive assessment. Neurodevelopmental Therapy (NDT) and Sensory Integration Therapy (SIT) were used as the main intervention methods (Table 1). NDT has three subheadings which are used to promote motor function in children, while SIT aims to integrate the senses with movements [8].

The authors used some adjunctive treatments to the NDT and SIT in order to facilitate the motor development and to prevent possible deformities. Ankle-Foot Orthosis (AFO) was used to position foot and give sensorial stimuli to this region. At the 30th month, 25 right lumbar scoliosis was diagnosed and Boston corset was started to be used to prevent progression of this angle. Kinesio Tape (KT) was also used and was applied to facilitate hypotonic muscles of ankle and knee joints by the two authors who have certificates. Authors applied it once a week, for last 3 months of the PHTR.

\section{Results}

The GMFM results were presented in table 2. The most increase with around 50\% was provided in Section A during 2 years. While around $40 \%$ increase was acquired in Section B, small increases were observed in other sections.

Grasping skill gradually improved in 2 years. The most important increase was acquired in $\mathrm{T}_{1}-\mathrm{T}_{2}$ time interval (Table 2).

At the beginning of the treatment, he did not even hold his head. Following one year rehabilitation, the case could raise his head, sit dependently, and then sit independently. After the second year, kneeling, standing up and walking with support were acquired. Figures 1,2 show two pictures belonging to the child at $\mathrm{T}_{0}$ and $\mathrm{T}_{2}$.

Parent found difficult to use $\mathrm{AFO}$ and corset as the child was irritated while wearing them so a regular use could not be provided.

\section{Discussion}

This case report describes how a PHTR program affects impairments and activity in a child with PKS during two years. Data from this paper supports the use of various approaches for these children. When "Pallister Killian Syndrome" was searched in 


\begin{tabular}{|c|c|c|}
\hline \multicolumn{3}{|c|}{ Sensory Integration Therapy } \\
\hline Vestibular Sense & Eyesight & Auditory Sense \\
\hline $\begin{array}{l}\text {-swinging materials } \\
\text {-slide boards }\end{array}$ & $\begin{array}{c}\text {-Dark room and lights } \\
\text {-Various toys (particularly } \\
\text { colourful) }\end{array}$ & $\begin{array}{l}\text {-Voice of flowing water (the parent } \\
\text { detected his interest on this) } \\
\text {-Voice toys }\end{array}$ \\
\hline \multicolumn{3}{|c|}{ Neurodevelopmetal Therapy (BOBATH) } \\
\hline Stimulation & Communication & Facilitation \\
\hline $\begin{array}{l}\text {-Weight bearing and approximations } \\
\text { were used. } \\
\text {-classical massage for particularly } \\
\text { face and plantar regions; } \\
\text {-different soft materials like cotton to } \\
\text { environment of hypersensitive areas; } \\
\text {-make orders of the materials } \\
\text { according to hypo or hypersensitive } \\
\text { areas: cotton, hair felt, spiky massage } \\
\text { balls, sand, shaving foam, ball pit } \\
\text {-wrapping with duvet } \\
\text {-compression with pilates ball, } \\
\text {-massage with various hard materials } \\
\text { like massage balls, brushes, and } \\
\text { combs } \\
\text { - weighted vests were worn } \\
\text { * He was also taken to the standing } \\
\text { table in otder to verticalise for } 1 \text { hour } \\
\text { every day as a home program. } \\
\text { * Progression was provided by } \\
\text { approaching the mentioned stimuli to } \\
\text { the hypersensitive area or increasing } \\
\text { the duration of their application. }\end{array}$ & $\begin{array}{l}\text { - Family education was presented. } \\
\text { - Verbal stimuli was used during all } \\
\text { movements of the sessions. } \\
\text { - Eye tracking and eye contact was } \\
\text { tended to be improved moving his } \\
\text { loved topys and doing some PT } \\
\text { interventions in front of mirrors. }\end{array}$ & 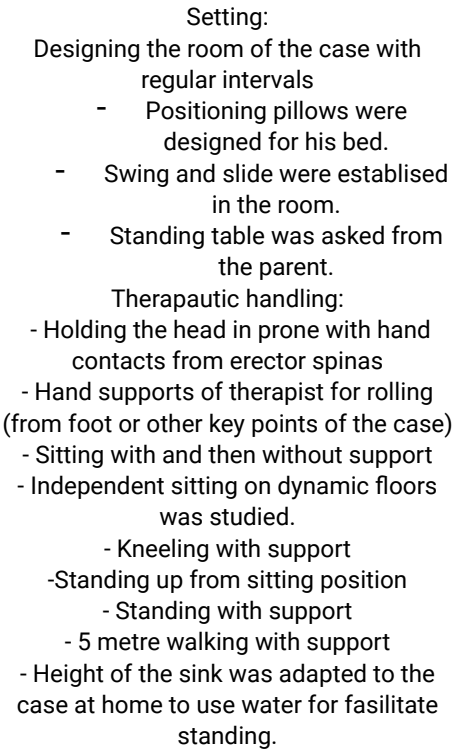 \\
\hline
\end{tabular}

Table 2: Results of the assessment of motor skills.

\begin{tabular}{|c|c|c|c|}
\hline GMFM & $\mathbf{T}_{\mathbf{0}}(\%)$ & $\mathbf{T}_{\mathbf{1}}(\%)$ & $\mathbf{T}_{\mathbf{2}}(\%)$ \\
\hline Section A & 35.29 & 80.39 & 84.31 \\
\hline Section B & 13.33 & 35 & 51.67 \\
\hline Section C & 0 & 7.14 & 7.14 \\
\hline Section D & 0 & 0 & 7.69 \\
\hline Section E & 0 & 0 & 2.78 \\
\hline Total & 9.72 & 24.51 & 30.72 \\
\hline Ping Pong Ball (sec) & 0 & 4 & $>10$ \\
\hline GMFM: Gross Motor Function Measure. & & & \\
\hline
\end{tabular}

Figure 1: The case at $\mathrm{T}_{0}$ and $\mathrm{T}_{2}$.

\begin{tabular}{l} 
Figure 1: The case at $\mathrm{T}_{0}$ and $\mathrm{T}_{2}$. \\
\hline Figure 2: The case at $\mathrm{T}_{0}$ and $\mathrm{T}_{2}$.
\end{tabular}


the literature, some authors presented surgical interventions and dental problems in these children [9,10], and hearing aids were found to be useful in a case report [11]. Multiple papers investigated the clinical features of PKS and the difficulties of its prenatal diagnosis $[12,13]$. Although PHTR for children with PKS was mentioned in some papers, no details about these interventions or their results were presented [2,11].

The case had normal birth weight and birth height and various authors stated that they may be normal, but then their growth slows down $[2,4,5]$. The case was followed up for 2 years bacause it was difficult to acquire essential changes in shorter time intervals. Similarly, various authors followed children with neurological diseases and genetic syndromes for years $[14,15]$. NDT and SIT which are commonly used in order to improve motor skills in the literature were the main treatment approaches for the case $[8,16]$. This is the first paper presenting a PHTR program and its long-term effects for this population.

The case had very severe hypotonia at the beginning so there was no resistance of his muscles against gravity. 90.9\% of the reported cases by Blyth et al. [1], were hypotonic in the neonatal period. In addition, looking at the results about gross and fine motor skills reported in the paper by Blyth et al. [1], it was seen that there was not a standard development in the ages of children with PKS. According to Blyth et al. [1], walking was achieved in most cases, but different walking ages were reported, for example, a child started to walk at 8 years old. This is contrary to general opinion as independent sitting at 2 years old may be shown as a predictor of walking by age 6-7 in the children with Cerebral Palsy [17]. It seems not possible to state this type of general predictions in this population so the therapists cannot mention any target to the parent. Moreover, our case generally showed progression, but very slow, in his motor development and this shows a parallel result to the paper by Blyth et al. [1]. On the other hand, Saito et al. [18], reported the adulthood of two cases with PKS and stated a regression in the motor skills of these cases that lost their ability to stay sitting or to rollover following adolescence. Therefore, further research is required to get evidence and to determine whether these children may catch a standard development with rehabilitation methods.

Our results showed that various NDT and SIT approaches can be used for children diagnosed with PKS. Similar to our paper, NDT was found to be effective to improve gross motor functions in a case report by Jeevanantham et al. [16,] Jung [19] also used the SIT for a case with Goldenhar Syndrome and found effective on tactile problems and developmental delay.

In addition to NDT and SIT, two adjunctive treatments, KT and orthoses, were used. As KT was applied as an adjunct treatment, no quantitative change could be assessed or observed. Nevertheless when the parent was asked about its effectiveness, they stated "it does not cause any negative effect and it may be effective so we can continue applying". Only one paper by Kamal-Eldeen et al. [20], investigated the effects of KT on hypotonic muscles of children with obstetric brachial plexus injuries and found it effective as an adjunct method to physiotherapy.

Moreover, scoliosis developed despite the PHTR intervention. There is a high risk of developing scoliosis in hypotonic cases. In a study [1], 27.3\% of children with PKS who were 1-14 years of age had scoliosis. Although corset and AFO had adequate evidence in various aims, parent found difficult to use them as the child was irritated while wearing them.

Children with PKS have various comorbidities and difficulties which may probably affect the PHTR ${ }^{1}$. Among them, our case had visual and mental problems. In addition, seizures as very commonly seen comorbidity in this population were not found in our case, but many authors reported that their onset age and even their types varied $[1,19]$. Nevertheless, it is considered that if he had this comorbidity, it could have negatively affected our progressing. 
This study presents the first attempt of representation of a PHTR program and its effects on motor skills of a child with PKS. Determination of the most effective intervention among the PHTR program has not been possible as they were all applied together. Further studies with higher quality research designs are required to assess more accurately the effects of the PHTR.

This case report presented a picture of therapy approaches for a child with PKS. The long-term effects of these interventions were performed on motor skills of the child. Since there is very limited information in the literature, this is the first paper suggesting a therapy program for children with PKS and discussing its results. Thus, this paper will guide for all health professionals working with children with PKS and PKS like genetic disorders.

\section{References}

1. Blyth $M$, Maloney V, Beal S, Collinson M, Huang S, et al. Pallister-Killian syndrome: a study of 22 British patients. J Med Genet. 2015; 52: 454-464. Ref.: https://goo.gl/eVyUQW

2. Stone L, Tripuraneni R, Bain M, Hernandez C. Pallister-Killian syndrome in a two-year-old boy. Clin case rep. 2017; 5: 774-777. Ref.: https://goo.gl/oZ9Ppc

3. Teschler-Nicola M, Killian W. Case report 72: Mental retardation, unusual facial appearance, abnormal hair. Synd Ident. 1981; 7: 6-7. Ref.: https://goo.gl/9jtsHF

4. Srinivasan A, Wright D. Pallister-Killian syndrome. The Am J Case Reports. 2014; 15: 194-198. Ref.: https://goo.gl/CY3KWp

5. Schinzel A. Tetrasomy 12p (Pallister-Killian syndrome). J Med Genet. 1991; 28: 122-125. Ref.: https://goo.gl/hGsNEJ

6. Russell DJ, Rosenbaum PL, Wright M. Clinics in Developmental Medicine: Gross Motor Function Measure (GMFM-66 and GMFM-88) User's Manual Section ed. 2013. Ref.: https://goo.gl/MdqRUP

7. Alaniz ML, Rosenberg SS, Beard NR, Rosario ER. The effectiveness of aquatic group therapy for improving water safety and social interactions in children with autism spectrum disorder: a pilot program. J Autism Dev Disord. 2017; 47: 4006-4017. Ref.: https://goo.gl/Z9GJvV

8. Shamsoddini A. Comparison between the effect of neurodevelopmental treatment and sensory integration therapy on gross motor function in children with cerebral palsy. Iran J Child Neurol. 2010; 4: 31-38. Ref.: https://goo.gl/EiYW5a

9. Didinen S, Atabek D, Kip G, Patır Münevveroğlu A, Tulunoğlu Ö. Dental Treatment of a Child with Pallister-Killian Syndrome. Case Rep Dent 2016; 4130961; 4. Ref.: https://goo.gl/zBVrJG

10. Baglaj M, King J, Carachi R. Pallister-Killian syndrome: a report of 2 cases and review of its surgical aspects. J Pediatr Surg. 2008; 43: 1218-1221. Ref.: https://goo.gl/A3fGTJ

11. Çiprut A, Akdaş F. Pallister-Killian syndrome: A case with sensorineural hearing loss. Int J Pediatr Otorhinolaryngol. 2006; 1: 238-242. Ref.: https://goo.gl/9hoL48

12. Filloux FM, Carey JC, Krantz ID, Ekstrand JJ, Candee MS. Occurrence and clinical features of epileptic and non-epileptic paroxysmal events in five children with Pallister-Killian syndrome. Eur $\mathrm{J}$ Med Genet. 2012; 55: 367-373. Ref.: https://goo.gl/dfxi4k

13. Cetin Z, Sanhal C, Karauzum SB, Mendilcioglu I, Yakut S. Ultrasonographic and Cytogenetic Issues in Prenatal Diagnosis of Pallister Killian Syndrome. Genet Couns. 2016; 27: 233-237. Ref.: https://goo.gl/Rq84uL

14. Lytsy P, Carlsson L, Anderzén I. Effectiveness of two vocational rehabilitation programmes in women with long-term sick leave due to pain syndrome or mental illness: 1-year follow-up of a randomized controlled trial. J Rehabil Med. 2017; 49: 170-177. Ref.: https://goo.gl/h9aMrr

15. Hovik KT, Plessen KJ, Cavanna AE, Skogli EW, Andersen PN, et al. Cognition, emotion and behavior in children with Tourette's syndrome and children with ADHD-combined subtype-a two-year follow-up study. PloS one. 2015; 10: e0144874. Ref.: https://goo.gl/py96bJ

16. Jeevanantham D, Nagooran GJ, Christopher SK. Effect of Neurodevelopmental therapy on tuberous sclerosis-A Case Report abstract. IJTRR. 2012; 1: 2-2. Ref.: https://goo.gl/V9sBZY

17. Wu YW, Day SM, Strauss DJ, Shavelle RM. Prognosis for ambulation in cerebral palsy: a populationbased study. Pediatrics. 2004; 114: 1264-1271. Ref.: https://goo.gl/XjR4ka 
18. Saito Y, Masuko K, Kaneko K, Chikumaru Y, Saito K, et al. Brain MRI findings of older patients with Pallister-Killian syndrome. Brain Dev. 2006; 28: 34-38. Ref.: https://goo.gl/ua1369

19. Jung HS. Sensory Integration therapy Evaluation and Intervention in Clients with Goldenhar Syndrome: A Case Report. J Korea Cont Assoc. 2011; 11: 286-295. Ref.: https://goo.gl/g1npNX

20. Kamal-Eldeen RS, Awooda HA, El-Maksoud GMA. Effectiveness of Kinesio Tape on Wrist Extensor Muscles in Children with Obstetric Brachial Plexus Injuries. J Appl Life Sci Int. 2016; 7: 1-7. Ref.: https://goo.gl/3nuzP8 